\title{
La inteligencia artificial en la Educación Física en tiempo de COVID 19
}

\author{
Artificial intelligence in physical education in time COVID-19 \\ Inteligência artificial na educação física no tempo COVID-19
}

Marco Antonio Toala Pilay

toala.marco@unesum.edu.ec

https://orcid.org/0000-0002-6034-5719

Universidad Estatal de Sur de Manabí, Manabí-Ecuador

Universidad Nacional del Rosario, Rosario-Argentinaz

Vicente Fray Romero Castro

vicente.romero@unesum.edu.ec

https://orcid.org/0000-0001-5792-0105

Universidad Estatal de Sur de Manabí, Manabí-Ecuador
Martha Irene Romero Castro

martha.romero@unesum.edu.ec

https://orcid.org/0000-0001-5043-8295

Universidad Estatal de Sur de Manabí, Manabí-Ecuador

Rosario Magdalena Romero Castro
rosario.romero@unesum.edu.ec
https://orcid.org/0000-0002-6016-9145

Universidad Estatal de Sur de Manabí, Manabí-Ecuador

Artículo recibido 3 de septiembre 2021, arbitrado y aceptado 7 de octubre 2021 y publicado 20 de diciembre 2021

\section{RESUMEN}

La Inteligencia Artificial, al igual que otras tecnologías disruptivas, son hoy un espacio de innovación para la educación en época del COVID. Este estudio tributo al proyecto de investigación de la universidad estatal del sur de Manabí: la enseñanza constructivista sustentado en la inteligencia artificial. Determinar el enfrentamiento de alumnos y profesores, las clases de Educación Física en aislamiento. Se realizó un estudio descriptivo y transversal en 7 profesores y 28 estudiantes, se manejaron algunas variables: metodología física del trabajo de educación a distancia, situación de aislamiento pandémico. $75 \%$ estudiantes señalaron la eficiencia de las capsulas educativas más didáctica que las guías educativas, el $100 \%$ profesores el refieren lo difícil de la utilización de las clases online, pero a la vez son muy motivadoras. Las educaciones físicas virtuales se han convertido en herramientas eficaces que pueden contribuir a la calidad de vida de las personas aisladas.

Palabras clave: Inteligencia artificial; Pandemia COVID-19; Educación Física; TIC; entorno virtual

ABSTRACT

RESUMO

Artificial Intelligence, like other disruptive technologies, is today a space of innovation for education in the COVID era. This study tribute to the research project of the state university of southern Manabi: sustained in artificial intelligence. To determine the confrontation of students and teachers, Physical Education classes in isolation. A descriptive and transversal study was carried out in 7 teachers and 28 students, some variables were handled: physical methodology of distance education work, pandemic isolation situation. $75 \%$ of the students pointed out the efficiency of the educational capsules more didactic than the educational guides, $100 \%$ of the teachers referred to the difficulty of using online classes, but at the same time they are very motivating. Virtual physical education has become an effective tool that can contribute to the quality of life of isolated people.

Key words: Artificial intelligence; Pandemic COVID-19; Physical education, ICT, Virtual environment
A Inteligência Artificial, tal como outras tecnologias disruptivas, é hoje um espaço de inovação para a educação na era da COVID. Este estudo tributo ao projecto de investigação da universidade estatal do sul de Manabí: sustentado em inteligência artificial. Determinar o confronto de estudantes e professores, aulas de Educação Física isoladas. Foi realizado um estudo descritivo e transversal com 7 professores e 28 alunos, foram utilizadas algumas variáveis: metodologia física do trabalho de educação à distância, situação de isolamento pandémico. 75\% dos alunos apontaram a eficiência das cápsulas educativas mais didácticas do que os guias educativos, $100 \%$ dos professores referiram a dificuldade de utilizar as aulas em linha, mas ao mesmo tempo são muito motivadores. A educação física virtual tornou-se um instrumento eficaz que pode contribuir para a qualidade de vida das pessoas isoladas.

Palavras-chave: Inteligência artificial; COVID- 19 pandemia; Educação física; TIC; ambiente virtual 


\section{INTRODUCCIÓN}

Una nueva ola de desarrollos tecnológicos se está moviendo hacia la inteligencia artificial (IA). Esta "fuerza poderosa" está respaldada por avances en realidad aumentada, reconocimiento de voz y emociones, algoritmos de construcción de máquinas y plataformas digitales, aprendizaje avanzado y gestión de datos (Crawford et al., 2019).

La inteligencia artificial desarrolla la vida, interacción, ambiental Floridi et al., (2018) social, cultural, económica, ambiental y ética. Aporta a la misma persona en aspectos como la memoria, los procesos cognitivos. longevidad, capacidad física e intelectual para designar y reproducir estados y atributos propios (Marín-Casanova, 2018; González, 2018). Estos desarrollos perfilan la reestructuración de la cultura humanista como marco para repensar la subjetividad, "el horizonte del conocimiento humano" y "el hombre mismo", más allá de la "recultura significativa"(MarínCasanova, 2018).

La Inteligencia Artificial proyecta desafíos a disciplinas como la Antropología en cuestiones de la condición humana (García-Gutiérrez, 2017), Psicología relacionada con la psicoterapia y la transformación conductual (Rivera y Sánchez, 2018), origen espiritual y significado del hombre (Kotze, 2018), cuidado de la salud, diagnóstico, tratamiento clínico, Educador, y la relación entre los medios de comunicación y los medios de comunicación, sus aspectos profesionales y éticos (Salazar, 2018; Barrios et al., 2019).

Es de observación y análisis que en medio de la pandemia de COVID-19, las diferentes propuestas de investigación han manejado su atención en las necesidades esenciales de la enseñanzaaprendizaje en línea en la educación los riesgos y desventajas de estas tendencias tecnológicas desde la perspectiva sostenible. La oportunidad transformadora para la investigación en educación debido a los nuevos procesos de pensamiento planteados por la pandemia, identificando las mejores prácticas derivadas de la literatura de las medidas de implementación de la transformación digital; especialmente en conocer la perspectiva de los estudiantes sobre el uso de las tecnologías para comunicarse con sus profesores.

La Emergencia Sanitaria Pandémica Global COVID 2020 ha introducido una serie de acciones que se han desencadenado como una nueva solución para la contención en todo el mundo. El sector de la educación está cambiando drásticamente de cara a la virtualidad, creando oportunidades que necesitan ser analizadas y evaluadas para hacer que la educación virtual sea sostenible y mitigar los riesgosidentificados. Las tecnologías de inteligencia, especialmente las aplicaciones de inteligencia artificial (IA), se integran como opciones para incrementar la productividad de los diversos procesos que implementan estas aplicaciones en la educación superior.

Ecuador no cuenta con una estrategia de inteligencia artificial (IA), para mantener la calidad del proceso de educación-aprendizaje de conocer la educación superior, buenas prácticas, oportunidades y evaluar riesgos durante el COVID, en varios países de la región se están desarrollando aplicaciones, pero aún se está analizando la importancia de trabajar en conjunto los esfuerzos de desarrollo de la IA con la participación del gobierno, la academia, la industria y la sociedad civil (Gómez et al., 2020).

Siguiendo la línea de pensamiento en el ecuador las condiciones del confinamiento retrasaron el inicio del año escolar 2020-2021 en las zonas 
costeras, pero dos meses después del inicio de las clases en modalidad virtual, este escenario es para docentes y estudiantes como actores protagonista en la utilización de las tecnología de la información saliendo del ambiente tradicional de las clases de Educación Física (Alfonzo et al., 2020).

El Plan Educativo COVID-19, también llamado proceso educativo en el contexto de la emergencia sanitaria, implementado por el Ministerio de Educación de Ecuador, fue creado para garantizar la continuidad educativa por medio de recursos de carácter pedagógico, metodológico y de contención emocional, contextualizados a las diferentes necesidades educativas de la población ecuatoriana en medio de la crisis sanitaria. (Ministerio de Educación, República de Ecuador, 2020).

A través del plan educativo la primera fase: Aprendemos juntos en casa y los reguladores educativos ecuatorianos continúan el proceso educativo. Brinda lineamientos y recomendaciones, y decide enviar asignaciones en línea. Muestra el proyecto en una carpeta de caja, preferiblemente en formato digital. Este formato requiere que los estudiantes dirijan el aprendizaje y los docentes controlan y guía el proceso para abordar la seguridad, salud física y mental del estudiante.

Además del llenado de formulario educativo para obtener más detalles sobre el nombre del proyecto, audiencia, mecanismo de evaluación, en la segunda fase la segunda fase se empezó a implementar una vez que concluyó el Decreto de renovación del estado de excepción provocado por la crisis pandémica por lo que las instituciones educativas elaboraron planes de continuidad educativa y retorno progresivo a las aulas, para poder implementar modalidades de estudio semipresencial, cumpliendo los protocolos de bioseguridad establecidos por el Centro de Operaciones de Emergencias [COE], la tercera fase este proceso educativo se orienta la utilización del Currículo Priorizado, destinado a las instituciones educativas que disponen de plataformas virtuales para garantizar la continuidad educativa y cuyos estudiantes cuenten con conectividad y dispositivos electrónicos para la interacción educativa; y el Currículo Priorizado para la Emergencia, destinado a las instituciones educativas que no disponen de plataformas virtuales o cuyos estudiantes no tengan conectividad o dispositivos electrónicos para poder continuar con un proceso educativo en línea; todo esto con el objetivo de garantizar el nivel de logro 1 de los estándares de aprendizaje, para que todos los estudiantes del país tengan las mismas oportunidades de aprendizaje. (Ministerio de Educación. República de Ecuador, 2020).

En este sentido, la crisis provocada por COVID-19 ha afectado a todos los servicios deportivos: competiciones a todos los niveles, programas y eventos deportivos, industria del fitness, turismo activo, Educación Física en la escuela (EF), actividades extraescolares, campos deportivos, deportes sociales y de salud, servicios sociales, deporte no organizado, formación basada en el aprendizaje presencial, etc. Afectó a todos los grupos de interés: todo tipo de deportistas, emprendedores, profesionales y técnicos, empleados o autónomos y trabajadores relacionados con el sector. Zona deportiva. También significa barreras de acceso y uso de soporte físico: espacios, estructuras, equipos y materiales (Gambau, 2020).

La Educación Física juega un papel importante, ya que es la única asignatura que puede reducir 
la ansiedad y el estrés en los estudiantes durante la cuarentena forzada. En este sentido, se considera que el aislamiento es la causa del estrés, los cambios emocionales y la disminución del rendimiento deportivo, y la actividad física en el hogar es la solución. Además, se cree que el arresto domiciliario ha causado efectos en la salud mental, como depresión y estrés. Y para prevenir estas enfermedades, hacen de la actividad física una de las soluciones (Posso et al., 2021).

Una de las áreas que más contribuyó durante y después de la cuarentena a la IA fue la Educación Física, es justo señalar que las aulas virtuales son el medio principal para asegurar la continuidad del proceso de enseñanza y aprendizaje, lo que hace que la investigación en Educación Física sea un desafío para los docentes. Los estudiantes de una institución determinan cómo se comparan los estudiantes y sus maestros. El problema radica es en la realización de clases de salud y Educación Física de forma independiente con COVID19. En base al panorama actual relacionado con la pandemia exige hacer los procesos de manera más rápida y pasar a construir soluciones.

Hechas las consideraciones anteriores, la investigación tuvo como con el objetivo determinar cómo ha enfrentado la población escolar y sus profesores, la problemática de realizar clases de
Educación Física en aislamiento por COVID-19, dándole salida al proyecto de la Universidad Estatal del Sur de Manabí acerca de la enseñanza constructiva sustentada en la inteligencia artificial.

\section{MÉTODO}

Se realizó un estudio descriptivo transversal en el distrito de educación 13D03 con el objetivo de determinar cómo ha enfrentado la población escolar y sus profesores, la problemática de realizar clases de Educación Física en aislamiento por COVID-19. El estudio fue exploratorio y corresponde a un método cuantitativo y cualitativo basado en un diseño de estudio aplicado a una muestra no aleatorizada de 7 profesores de EF y 28 alumnos. La técnica e instrumento usado fue la encuesta diseñada por grupos de experto con el propósito de recopilar información. Inicialmente, el estudio fue diseñado y construido sobre un marco lógico. El segundo paso fue verificar la evaluación de expertos y el tercer paso es aplicar la encuesta al muestreo piloto para determinar la confiabilidad. Finalmente, se realizó un análisis estadístico mediante el Alfa de Crombach y un análisis descriptivo. Se consideraron varias variables de interés: metodología física del trabajo de educación a distancia, situación de aislamiento pandémico COVID 19" y nueva Educación Física (Tabla 1).

Tabla 1. Operacionalización de la variable.

\begin{tabular}{lll}
\hline \multicolumn{1}{c}{ Variable } & \multicolumn{1}{c}{ Dimensiones } & Indicadores \\
\hline $\begin{array}{l}\text { Metodología física del trabajo de } \\
\text { educación a distancia }\end{array}$ & Recursos didácticos & Cápsulas educativas guías de aprendizaje. \\
& Etapas de una clase & Inicio, desarrollo y cierre. \\
& Plataformas de streaming & Zoom, Google meet, Instagram. \\
\hline
\end{tabular}




\begin{tabular}{lll}
\hline \multicolumn{1}{c}{ Variable } & \multicolumn{1}{c}{ Dimensiones } & \multicolumn{1}{c}{ Indicadores } \\
\hline $\begin{array}{l}\text { Contexto de aislamiento } \\
\text { por pandemia COVID-19 }\end{array}$ & $\begin{array}{l}\text { Personal learning networks } \\
\text { Salud mental }\end{array}$ & $\begin{array}{l}\text { Avances en nuevas generaciones }(\mathrm{Z}) . \\
\text { Estrés. }\end{array}$ \\
& Localización de residencia & Conectividad y modalidades Sincrónicas \\
Nueva Educación Física & Tareas online & o Asincrónicas de clases online. \\
& & Videos, guías, capsulas, Disertaciones \\
\end{tabular}

Para la recopilación de los datos se realizó en línea mediante el Software Google Forms, que permitió recoger la información de los usuarios encuestados. En este caso, todo se exporto a Excel para que los datos fuesen analizados estadísticamente. La información se procesó mediante el programa estadístico SPSS versión 21.0. Se utilizó el porcentaje como medida de resumen.

\section{RESULTADOS}

En el Gráfico 1 se muestra en materias de Educación Física, la dimensión recurso didáctico y como indicador las cápsulas educativas (videos) son más eficientes para captar su atención y más didácticas que las guías educativas, el 21 (75 \%) de los alumnos señalaron la eficiencia de las capsulas educativas y más didáctica que las guías educativas, el 7 (25 \%) no estuvieron de acuerdo, para su análisis se tuvieron en cuenta algunos aspectos como la visualización repetidas de los contenidos y la información de procesos de comprensión, posee un mecanismo alternativo para cuando no sea posible asistir a clase o simplemente para ponerse al día en el contenido del curso, permite repasar los contenidos, particularmente, cuando son introducidos temas de difícil comprensión, no así las guías educativas que son un documento de carácter instructivo y orientador.

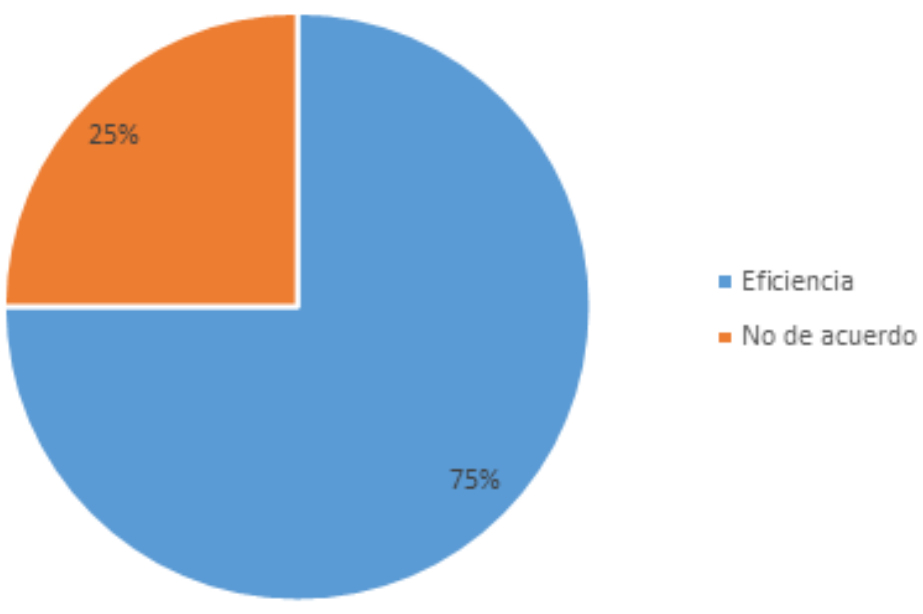

Gráfico 1. Recurso didáctico. 
Respecto a la estructura de la clase online (inicio-desarrollo-cierre) están presente, el 82, 1\% (23) respondieron afirmativamente mientras el restante $5(17.8 \%)$ señalaron que no siempre. El $96.4 \%$ (27), de los estudiantes en esta situación anormal (pandemia) le es muy difícil completar la tarea, ir a clase y participar.

Con respecto al componente motivacional se tuvieron en cuenta la parte afectiva y emocional para los cursos en línea, el 96,4\% (27) refiere que los cursos en línea fueron motivadores y solamente 1 (3.5\%) indico que los cursos en línea no son motivadores legítimo. Del total de alumnos, 67.8\% (19) opinaron afirmativamente la adaptación de la asignatura de Educación Física de modo correcto a la situación actual y el 32,14(9) manifestaron negativamente (Gráfico 2).

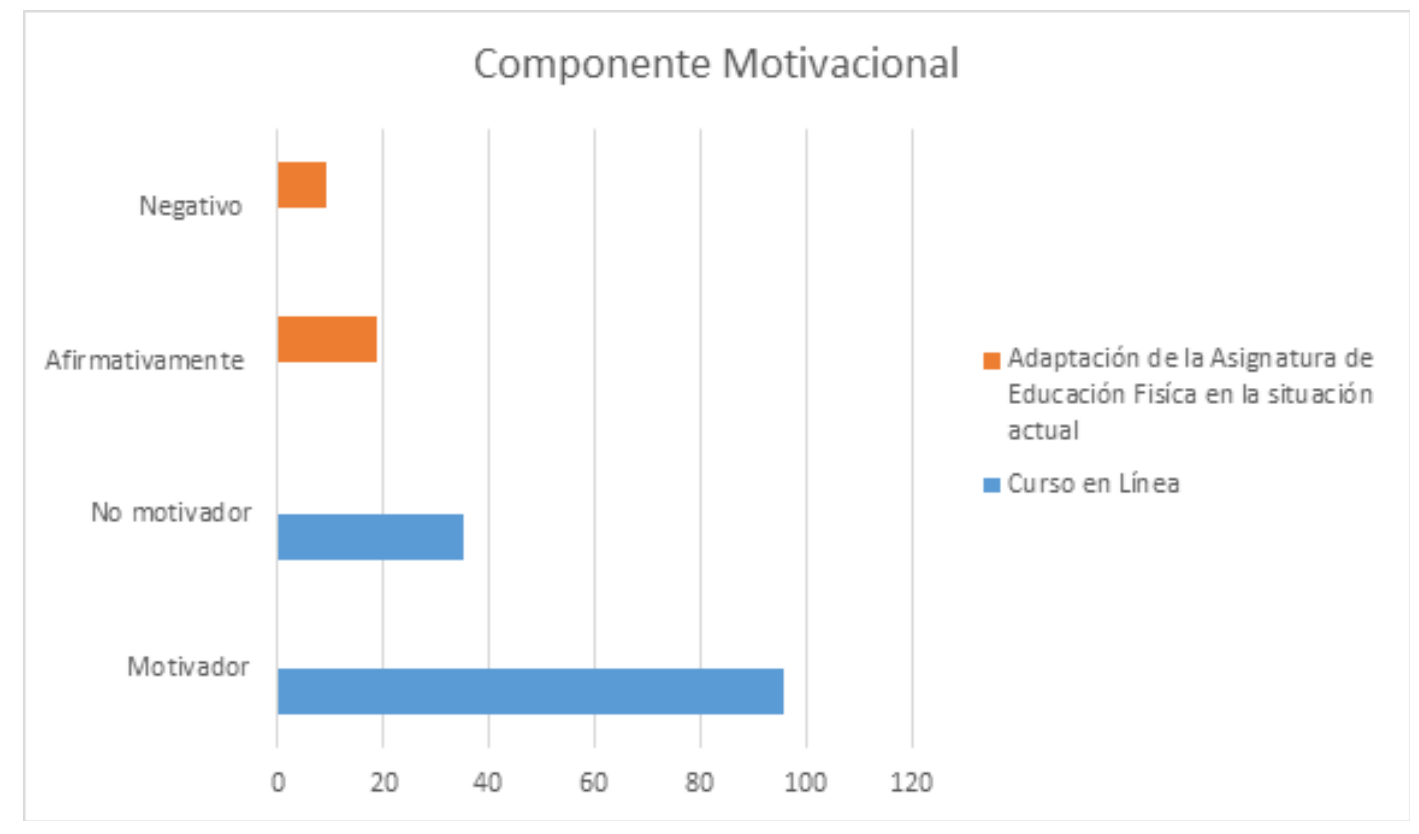

Gráfico 1. Componente motivacional.

A pesar que la mayoría de los estudiantes indicaron que le fue difícil adaptarse a la nueva metodología de enseñanza online, el 57.1\% (16) son capaces de participar en diferentes plataformas virtuales. (Zoom, Teams, Google Meet, etc.) 35.7 \%
(10) refirieron presentar dificultades, y solamente el 7,1\% (2) no la saben utilizar por las dificultades existente de la conectividad como elemento imprescindible para la actividad de la inteligencia artificial y más en tiempo de pandemia Gráfico 3. 


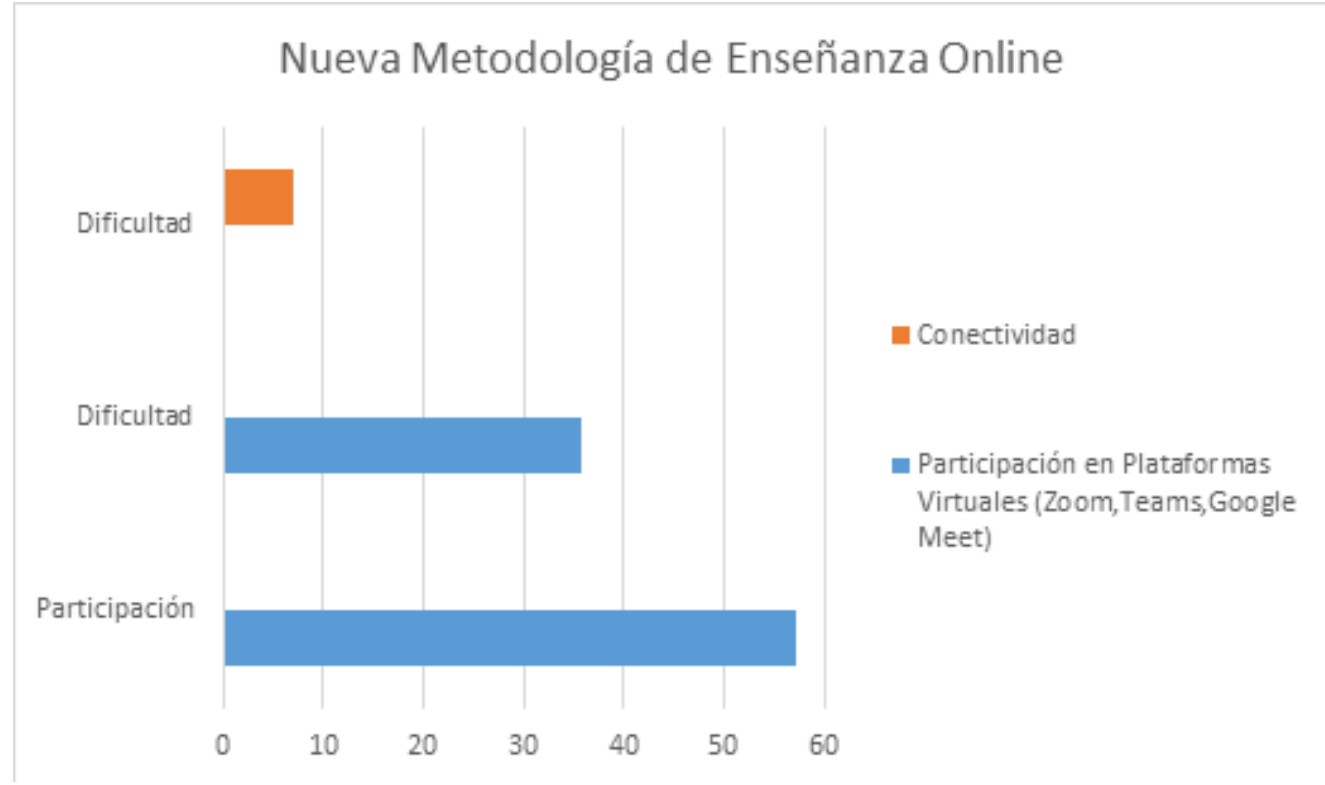

Gráfico 3. Nueva metodología de enseñanza online.

En estos tiempos de pandemia por la COVID19 se debe hacer frente a una situación inédita en la sociedad, donde, además de tener que afrontar los efectos directos del virus, como pueden ser la sintomatología de la enfermedad, el miedo al contagio y la preocupación por los seres queridos, también se debe lidiar con las medidas de cuarentena para frenar la pandemia, el 78.5\% (22) manifestaron sentirse estresado por lo que ha repercutido en su desempeño en clases de Educación Física.

En estos tiempos de pandemia donde los estudiantes y docentes se encuentran separados en tiempo y distancia la modalidad asincrónica se manifiesta que es la más utilizada.

Al menos la mitad 25 (89.2\%) de los alumnos han hecho uso de alguna aplicación de celular para ayudarse en su entrenamiento físico.

Los profesores consideran que las cápsulas didácticas son un recurso más eficaz debido a las entradas sobre el uso de guías de aprendizaje. Además, afirman que la estructura de clases de los cursos en línea (inicio, desarrollo, finalización) aún no se ha perdido. Cuando se trata de trabajar de forma remota, aunque se sientan capacitados para ser profesores en modo online, lo encuentran más complicado. De manera similar, afirmaron que los estudiantes con conocimientos de informática les facilitaron trabajar como profesores en línea. Los profesores también dicen que su institución los apoya en la formación técnica en línea. Los maestros se oponen firmemente a las lecciones presenciales porque son tan efectivas como las lecciones en línea. Por último, no indican ningún criterio con que los apoderados colaboren con la labor docente al responder correos, regresar materiales solicitados, etc.

La totalidad de los profesores refieren que le fue difícil la utilización de las clases online, pero a la vez son muy motivadoras. Con respecto a que la priorización Curricular ha sido de ayuda para su labor docente contexto de pandemia la mayoría de los profesores su respuesta fue afirmativa $5(71,4 \%)$. 
Todos los sujetos coincidieron en que menos espacio afectaría su motivación para realizar el curso. Además, aprecian cambios físicos debido al comportamiento sedentario de los estudiantes. En términos de modalidad de lección, las lecciones sincrónicas son más efectivas que las lecciones asincrónicas, y la mayoría de los profesores están de acuerdo en que RR.SS es más beneficioso en términos de difusión de información. Los profesores no están de acuerdo en que esta nueva forma de trabajar en línea tenga prioridad sobre el trabajo normal (presencial).

Estos profesores sintieron algún impacto en su salud mental 4 (57, $1 \%)$. Se manifiesta por parte del profesorado encuestado se han encontrado con muchas y diversas realidades de las familias del alumnado; familias pasando por problemas de salud (han pasado la COVID -19 o han sufrido perdida familiar) familias que han perdido el puesto de trabajo y tiene verdaderos problemas económicos, familias con poco recursos y conocimientos tecnológicos, y familia que no presentan ningunas de estas situaciones, frente a este escenario el docente expresa la necesidad de la búsqueda de una metodología adecuada para poner en función la Educación Física en estos tiempos de pandemia.

\section{Discusión}

El desarrollo tecnológico no es el mismo en todos los países y regiones, pero es imperativo implementar alternativas para mejorar la Educación Física con áreas remotas. La pandemia ha obligado a la educación deportiva a repensar sus métodos y objetivos, tanto en la escuela como en la universidad. ¿Cómo transmitir los contenidos de la Educación Física? ¿Qué soporte (estructurado, reposición, reutilización, nuevo) se puede utilizar para realizar un curso en un aula virtual? ¿Cómo entrenar habilidades deportivas en la escuela o la universidad? ¿Qué métodos debo utilizar para evaluar el rendimiento de los estudiantes y los atletas? Sin duda, la crisis pandémica ha hecho que la Educación Física sea comparable a lo que era hace meses. Parece ser lo más lejano que puede existir al final del concepto básico de aplicación de aprendizaje a distancia. variantes del aprendizaje en línea y la educación virtual (Isidori, 2020).

Según Cifuentes (2020) el aprendizaje a distancia requiere que estudiantes y profesores adopten un enfoque completamente diferente de las lecciones impartidas en persona. Los estudiantes y muchos profesores tienen poca o ninguna experiencia con esta forma de educación. Algunos maestros piensan que pueden usar los mismos métodos de enseñanza y aprendizaje que la enseñanza presencial, pero esto es un concepto erróneo.

Los resultados del estudio son similares a la investigación realizada por Bocaz et al., (2021) en relación a las variables vinculadas con la motivación de la utilización de la inteligencia artificia en el proceso de enseñanza y aprendizaje de la Educación Física en tiempos de pandemia así mismo la competencia de alumnos y profesores en la utilización de las TIC.

En esta ocasión, los docentes se enfrentan a una situación que cambia rápidamente y que requiere habilidades más profundas y un mejor dominio de las TIC. Conservar la motivación e interés de los alumnos e invitarlos a convertirse en protagonistas de su investigación (Villafuerte et al., 2020).

Es un aporte valioso de Parra (2020) donde apunta quelos profesoresy estudiantes deben abordar temas importantes, reconocer la responsabilidad y ser independientes en el aprendizaje. 
La Educación Física se caracteriza por priorizar la práctica como un medio para lograr resultados de aprendizaje, y argumentan que la situación actual es confusa para la práctica. Preste más atención a los cronómetros y los activos digitales.

Estudios como el de Alfonzo et al., (2020) están relacionados con el tema y los resultados son similares a este estudio. El autor menciona que "Los estudiantes también tienen problemas para administrar la plataforma”. Ralentiza el proceso. Otro aspecto es que no todo el mundo tiene una computadora o una computadora portátil, sino un teléfono inteligente o una tableta. Algunas personas lo usan, otras no. cámara o micrófono. Esto dificulta el desarrollo de la clase.

Es saludable acotar que tan importante es establecer un proyecto de actividades para la Educación Física virtual en tiempo de cuarentena, como también sostener la dinámica de estas actividades cuando ya esa cuarentena se haya retirado y se vuelva a la normalidad. En todo esto es fundamental preparar a la población para el futuro venidero que debe y tiene que ser mejor.

En ese mismo orden y dirección la Educación Física virtual debe tener como objetivo no solo potenciar las capacidades físicas sino fortalecer el pensamiento, la forma de proceder y operaren tiempo post COVID-19 que visto desde sus características tiene que depender de la habilidad en que se es capaz de resolver los problemas con la inteligencia, acotando a este pensamiento.

Se considera que determinadas situaciones requieren medidas especiales y una flexibilidad especial, que puede maximizar el potencial del estudiante.

\section{CONCLUSIONES}

La población escolar y sus profesores han enfrentado las clases de Educación Física en tiempos de COVID - 19 con las herramientas dela inteligencia artificial como capsulas educativas (videos), se ha trabajado en el componente motivacional y la utilización de las plataformas virtuales.

La pedagogía de la Educación Física debe estar dirigida a nuevas formas y estrategias para llegar mejor a más personas.

\section{REFERENCIAS}

Alfonzo, A., Caro, L., y Alcívar, L. (2020). Estrategias didácticas para la efectividad de la Educación Física: un reto en tiempos de confinamiento. Revista Electrónica Formación y Calidad Educativa (REFCalE), 8(3), 191-206. http:// refcale.uleam.edu.ec/index.php/refcale/article/ download/3260/2059

Barrios, H., Díaz, V., y Guerra, Y. (2019). Artificial Intelligence and Education,Challenges and Disadvantages for the Teacher. ARCTIC Journal, 72(12), 30-50. https://www.researchgate.net/ publication $/ 338236746 \% 0 \mathrm{D}$

Bocaz, F., Delgado, F., y Inzunza, V. (2021).Educación Física y salud bajo confinamiento covid-19 en profesores $y$ escolares de enseñanza media [Universidad De Concepción .Chile]. http:// repositorio.udec.cl/bitstream/11594/6053/1/ TESIS EDUCACIÓN FÍSICA Y SALUD BAJO CONFINAMIENTO.Image.Marked.pdf

Cifuentes, J. (2020). Docencia online y Covid-19: la necesidad de reinventarse. Revista de Estilos de Aprendizaje, 13(Especial), 115-127. http:// revistaestilosdeaprendizaje.com/article/ view/2149

Crawford, K., Dobbe, R., Dryer, T., Fried, G., Green, B., Kaziunas, E., Kak, A., Mathur, V., McElroy, E., Sánchez, A., Raji, D., Rankin, J., Richardson, R., Schultz, J., West, S., \& Whittaker, M. (2019). 
AI Now 2019 Report. https://ainowinstitute.org/ AI_Now_2019_Report.pdf

Floridi, L., Cowls, J., Beltrametti, M., Chatila, R., Chazerand, P., Dignum, V., Luetge, C., Madelin, R., Pagallo, U., Rossi, F., Schafer, B., Valcke, P., \& Vayena, E. (2018). AI4PeopleAn Ethical Framework for a Good AI Society: Opportunities, Risks, Principles, and Recommendations. Minds and Machines, 28(4), 689-707. https://doi.org/10.1007/s11023-0189482-5. Epub 2018 Nov 26

Gambau, V. (2020). COVID-19: La crisis ha afectado a todos. Revista Española de Educación Física y Deportes, 429(27), 15-28. https://www.reefd.es/ index.php/reefd/article/view/896/742

García-Gutiérrez, J. (2017). ¿Cómo mejorar al ser humano? Un análisis de las Pedagógica, tecnologías convergentes desde la antropología. Pedagogia e Vita, 75, 94-105. https://www. researchgate.net/publication/318653380\%0D

Gómez, C., Del Pozo, C., Martínez, C., y Martín del Campo, A. (2020). LA INTELIGENCIA ARTIFICIAL AL SERVICIO DEL BIEN SOCIAL EN AMÉRICA LATINA Y EL CARIBE: panorámica regional e instantáneas de doce países. BID Banco Interamericano de Desarrollo. https://doi.org/http://dx.doi. org/10.18235/0002393

González, F. (2018). La era de la perplejidad. Repensar el mundo que conocíamos. OPEN MIND. Fundación BBVA. https://www.bbvaopenmind. com/wp-content/uploads/2018/01/BBVAOpenMind-La-era-de-la-perplejidad-repensarel-mundo-que-conociamos.pdf

Isidori, E. (2020). Sports Pedagogy at the Time of COVID-19. Cultura, Ciencia y Deporte, 15(44), 145-146. https://doi.org/doi.org/10.12800/ccd. v15i44.1480
Kotze, M. (2018). The theological ethics of human enhancement: Genetic engineering,robotics and nanotechnology'. In die Skriflig, 52(3), 1-8. https://doi.org/dx.doi.org/10.4102/ids. v52i3.2323

Marín-Casanova, J. (2018). La resemantización TIC de la cultura humanista. index.Comunicación, 8(1), 179-195

Ministerio de Educación.República de Ecuador . (2020). Plan Educativo Aprendamos Juntos en Casa. https://educacion.gob.ec/plan-educativocovid-19-se-presento-el-16-de-marzo/

Parra, J. (2020). Prácticas de docencia tradicional en ambientes de educación virtual. Academia y Virtualidad. Academia y Virtualidad, 13(1), 93106. https://doi.org/https://doi.org/10.18359/ ravi.4295

Posso, R., Barba, L., Marcillo, J., \& Beltrán, S. (2021). Educación Física Interdisciplinaria ecuatoriana en el contexto dela covid-19. Accion, 17, 1-5. ttp://accion.uccfd.cu/index.php/accion/article/ view/154

Rivera, J., y Sánchez, D. (2018). Inteligencia artificial ¿Reemplazando al humano en la psicoterapia? Escritos, 24(53), 271-291. https://doi.org/doi. org/10.18566/escr.v24n53.a02

Salazar, I. (2018). Los robots y la Inteligencia Artificial. Nuevos retos del periodismo. Doxa Comunicación. Revista interdisciplinar de estudios de comunicación y ciencias sociales, 27, 295-315. https://doi.org/10.31921/doxacom. n27a 15

Villafuerte, J., Bello, J., Pantaleón, Y., y Bermello, J. (2020). Rol de Los docentes ante la Crisis del Covid-19, una mirada desde el enfoque humano. REFCalE, 8(1), 134-150. http:// refcale.uleam.edu.ec/index.php/refcale/article/ view/3214/1986 